\title{
Measurement of the Diffractive Deep-Inelastic Scattering Cross Section with a Leading Proton at HERA
}

\author{
Mikhail Kapishin (for the H1 Collaboration) * \\ JINR, Dubna, Russia \\ E-mail: kapishin@mail.desy.de
}

\begin{abstract}
Results are reported on a new measurement of the cross section for diffractive deep-inelastic scattering process $e p \rightarrow e X p$ with the leading final state proton detected in the H1 Forward Proton Spectrometer, using data collected at HERA-2. The cross section dependences on the proton fractional longitudinal momentum loss $x_{\mathbb{P}}$ and the squared four-momentum transfer at the proton vertex are interpreted in terms of an effective pomeron trajectory and a sub-leading exchange. The hypothesis of proton vertex factorisation is tested. The ratio of the diffractive to the inclusive $e p$ cross section is studied. The data are compared to QCD predictions at next-to-leading order based on parton distribution functions previously extracted from measurements of diffractive and inclusive deep-inelastic scattering.
\end{abstract}

XVIII International Workshop on Deep-Inelastic Scattering and Related Subjects, DIS 2010 April 19-23, 2010

Firenze, Italy

* Speaker. 


\section{Introduction}

Diffractive processes such as $e p \rightarrow e X p$ have been studied extensively in deep-inelastic electronproton scattering (DIS) at the HERA collider [1-4]. Their understanding at fundamental level is crucial for the development of quantum chromodynamics (QCD) at high parton densities. In a number of previous analyses, including [2], diffractive DIS events were selected on the basis of the presence of a large rapidity gap (LRG) between the leading proton and the remainder of the hadronic final state $X$. A complementary way to study diffractive processes is by direct measurement of the outgoing proton using Forward Proton Spectrometers (FPS) [1,3]. In contrast to the LRG case, the squared four-momentum transfer at the proton vertex $t$ can be reconstructed. The FPS also allows measurements up to higher values of the proton fractional longitudinal momentum loss $x_{I P}$ than is possible with the LRG method, extending into regions where the sub-leading trajectory is the dominant exchange. The FPS measurements provide a means of testing in detail whether the variables $x_{\mathbb{P}}$ and $t$ associated with the proton vertex can be factorised from the the variables $\beta=x / x_{\mathbb{P}}$ and $Q^{2}$ describing the hard interaction with the photon. Here $\beta$ is the longitudinal momentum fraction of the colour singlet carried by the struck quark, $x$ is the Bjorken scaling variable.

\section{The reduced cross section $\sigma_{r}^{D(4)}$ and test of proton vertex factorisation}

In this report, a new measurement of the reduced cross section $\sigma_{r}^{D(4)}\left(\beta, Q^{2}, x_{\mathbb{P}}, t\right)$ for the diffractive DIS process $e p \rightarrow e X p$ is presented, using the FPS data collected with the $\mathrm{H} 1$ detector at HERA-2. The reduced cross section is related to the diffractive structure functions $F_{2}^{D(4)}$ and $F_{L}^{D(4)}$ by

$$
\sigma_{r}^{D(4)}=F_{2}^{D(4)}-\frac{y^{2}}{1+(1-y)^{2}} F_{L}^{D(4)},
$$

where $y$ is the inelasticity. The reduced cross section is equal to the diffractive structure function $F_{2}^{D(4)}\left(\beta, Q^{2}, x_{\mathbb{P}}, t\right)$ to good approximation in the relatively low $y$ region covered by the current analysis. The analysed data sample corresponds to an integrated luminosity of $156 \mathrm{pb}^{-1}$. The data cover the range $0.1<|t|<0.7 \mathrm{GeV}^{2}, x_{\mathbb{P}}<0.1,4<Q^{2}<110 \mathrm{GeV}^{2}$ and $0.001<\beta<1$. The statistics of DIS

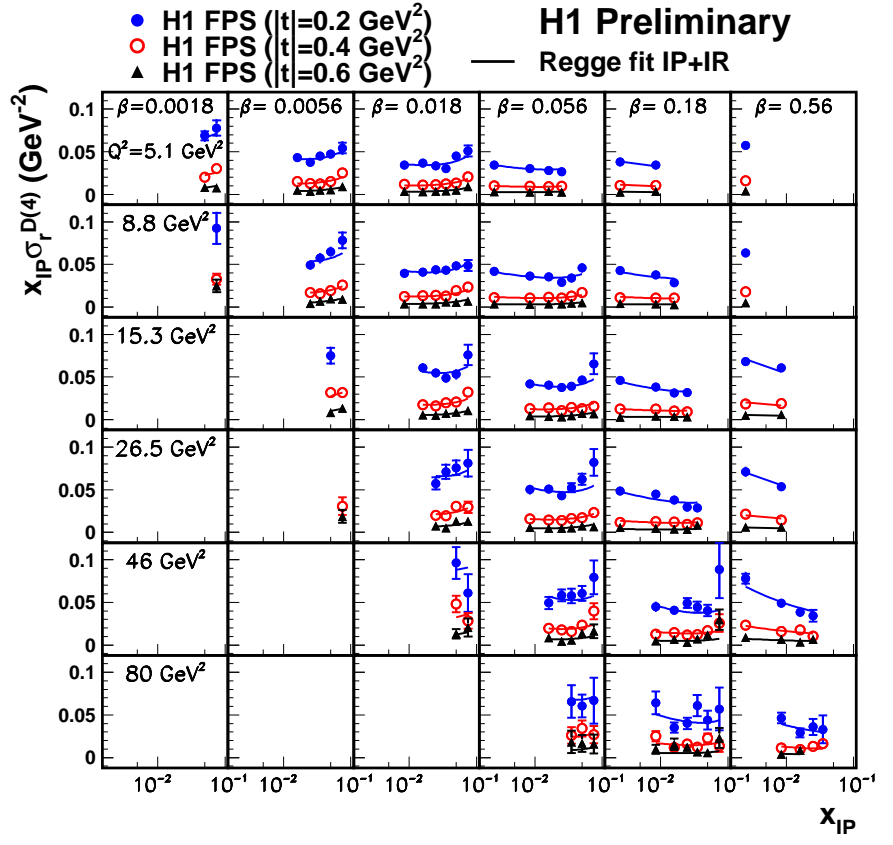

Figure 1: The diffractive reduced cross section, shown as a function of $x_{\mathbb{P}}$ for different values of $t, \beta$ and $Q^{2}$. The solid curves represent the results of the phenomenological 'Regge' fit to the data, including both pomeron $(\mathbb{P})$ and sub-leading $(\mathbb{R})$ trajectory exchange. 


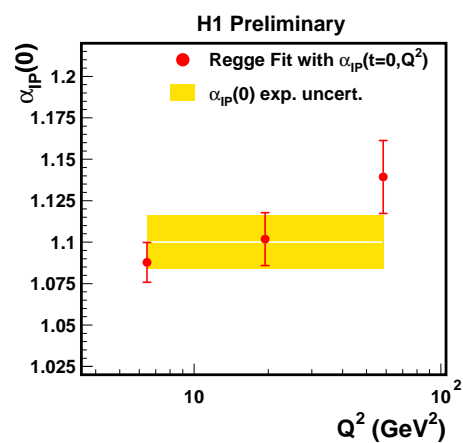

events with a leading proton are increased by a factor 20 compared to the previous H1 FPS analysis [1]. The kinematic range of the FPS measurement is extended to higher $Q^{2}$. Figure 1 shows $x_{\mathbb{P}} \sigma_{r}^{D(4)}$ as a function of $x_{\mathbb{P}}$ for different $t, \beta$ and $Q^{2}$ values. To describe the $x_{\mathbb{P}}$ and $t$ dependences quantitatively, the structure function $F_{2}^{D(4)}$ is parameterised by the form

$$
F_{2}^{D(4)}=f_{\mathbb{P}}\left(x_{\mathbb{P}}, t\right) \cdot F_{\mathbb{P}}\left(\beta, Q^{2}\right)+n_{\mathbb{R}} \cdot f_{\mathbb{R}}\left(x_{\mathbb{P}}, t\right) \cdot F_{\mathbb{R}}\left(\beta, Q^{2}\right) ; f_{\mathbb{P}, \mathbb{R}}\left(x_{\mathbb{P}}, t\right)=A_{\mathbb{P}, \mathbb{R}} \cdot \frac{e^{B_{\mathbb{P}, \mathbb{R}^{t}}}}{x_{\mathbb{P}} \alpha_{\mathbb{R}, \mathbb{R}^{(t)-1}}},
$$

which assumes a separate proton vertex factorisation of the $x_{\mathbb{P}}$ and $t$ dependences from those on $\beta$ and $Q^{2}$ for both the pomeron and a sub-leading exchange with no interference between the two contributions. The factors $f_{\mathbb{P}}$ and $f_{\mathbb{R}}$ correspond to flux factors for the exchanges and are taken from the Regge-motivated functions. The free parameters of the fit are the intercept and slope of the pomeron trajectory, $\alpha_{\mathbb{P}}(t)=\alpha_{\mathbb{P}}(0)+\alpha_{\mathbb{P}}^{\prime} t$, the exponential $t$-slope parameter $B_{\mathbb{P}}$, the normalisation coefficients $F_{\mathbb{P}}\left(\beta, Q^{2}\right)$ for the pomeron contribution at each of the $\left(\beta, Q^{2}\right)$ values considered, and the single parameter $n_{\mathbb{R}}$ describing the normalisation of the sub-leading exchange contribution. As in $[1,2]$, the normalisation coefficients $F_{\mathbb{R}}\left(\beta, Q^{2}\right)$ for the sub-leading exchange in each $\beta$ and $Q^{2}$ bin are taken from a parameterisation of the pion structure function [8].

The fit provides a good description of the $x_{\mathbb{P}}$ and $t$ dependences of the data. The result for $\alpha_{\mathbb{P}}(0) \simeq 1.10$ (figure 2 ) is compatible with that obtained from $\mathrm{H} 1$ data previously measured using the LRG and FPS methods [1,2] and with ZEUS measurements [3,4]. It is also consistent within uncertainties with the pomeron intercept describing soft hadronic scattering, $\alpha_{\mathbb{P}}(0) \simeq 1.08$ [6].

Figure 2: Results from the 'Regge' fit in which additional free parameters are included, corresponding to the values of $\alpha_{\mathbb{P}}(0), \alpha_{\mathbb{P}}^{\prime}$ and $B_{\mathbb{P}}$ in three different ranges of $Q^{2}$. The bands show the result and experimental uncertainty from the standard fit over the whole $Q^{2}$ range.
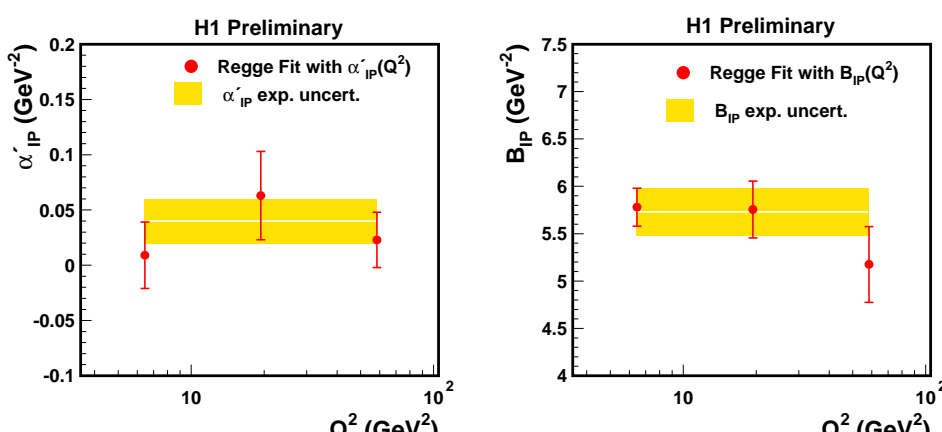

In a Regge approach with a single linear exchanged pomeron trajectory, $\alpha_{\mathbb{P}}(t)=\alpha_{\mathbb{P}}(0)+\alpha_{\mathbb{P}}^{\prime} t$, the exponential $t$-slope parameter $B$ of the diffractive cross section is expected to decrease logarithmically with increasing $x_{\mathbb{P}}$, an effect which is often referred to as 'shrinkage' of the diffractive peak. The degree of shrinkage depends on the slope of the pomeron trajectory, which is $\alpha_{\mathbb{P}}^{\prime} \simeq 0.25 \mathrm{GeV}^{-2}$ for soft hadron-hadron scattering at high energies. The FPS data favour a small value of $\alpha_{I P}^{\prime}$ (figure 2), as expected in perturbative models of the pomeron [7]. This result is inconsistent with the expected value of $\alpha_{\mathbb{P}}^{\prime}$ from soft hadron-hadron scattering. The results for $\alpha_{\mathbb{P}}^{\prime}$ and $B_{\mathbb{P}}$ are compatible with those obtained previously from the H1 and ZEUS data measured using the FPS detectors $[1,3]$. To check a possible breakdown of proton vertex factorisation implied by a dependence of the $\alpha_{\mathbb{P}}(0), \alpha_{\mathbb{P}}^{\prime}$ and $B_{\mathbb{P}}$ on $Q^{2}$, a modified version of the 'Regge' fit of the data is performed in 
three different ranges of $Q^{2}$. The results of the fits, shown in figure 2, indicate no strong dependence on $Q^{2}$.

The $t$-dependence of the cross section is parameterised by an exponential function such that $\mathrm{d} \sigma / \mathrm{d} t \propto e^{B t}$. Figure 3 shows the slope parameter $B$ as a function of $x_{\mathbb{P}}$ for data averaged over $Q^{2}$ and $\beta$. The results for $B$ are compared with a parameterisation of the $t$-dependence from the 'Regge' fit to $F_{2}^{D(4)}$. A good description of the data over the full $x_{\mathbb{P}}, Q^{2}$ and $\beta$ range in figure 3 confirms the quality of the fit. At low $x_{\mathbb{P}}$, the data are compatible with a constant slope parameter, $B \simeq 6 \mathrm{GeV}^{-2}$. No significant $Q^{2}$ or $\beta$ dependence of the slope parameter $B$ is observed for data points with $x_{\mathbb{P}} \leq 0.025$. The sub-leading exchange contribution integrated over this kinematic range is $7 \%$. A weak decrease of the slope $B$ from $6 \mathrm{GeV}^{-2}$ to below $5 \mathrm{GeV}^{-2}$ is observed towards larger values of $x_{\mathbb{P}}>0.05$, where the contribution from the sub-leading exchange is significant This reduction of the slope parameter indi-

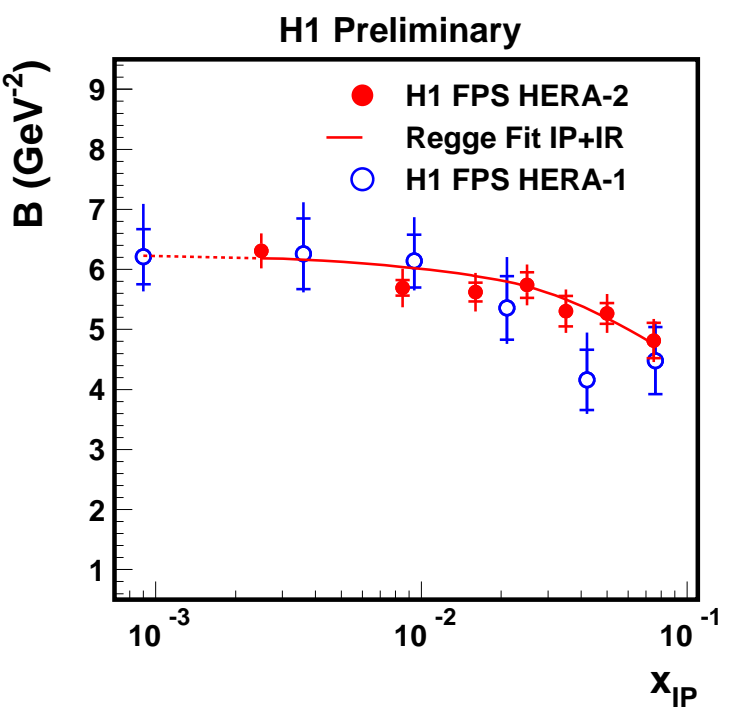

Figure 3: The slope parameter $B$ obtained from the fit of the form $\mathrm{d} \sigma / \mathrm{d} t \propto e^{B t}$ shown as a function of $x_{\mathbb{P}}$. The data are averaged over $Q^{2}$ and $\beta$. The solid curve represents the results of the phenomenological 'Regge' fit to the data, including both pomeron (IP) and sub-leading $(\mathbb{R})$ trajectory exchange. The previously published H1 FPS results [1] are also shown. cates that the size of the interaction region reduces for $\mathbb{R}$ exchange compared to $\mathbb{P}$.

\section{Comparison between Diffractive and Inclusive DIS}

By analogy with hadronic scattering, the diffractive and the total cross sections can be related via the generalisation of the optical theorem to virtual photon scattering [9]. Many models of low $x$ DIS assume links between these quantities $[10,11]$. Comparing the $Q^{2}$ and $x$ dynamics of the diffractive with the inclusive cross section is therefore a powerful means of developing our understanding of high energy QCD, comparing the properties of diffractive PDFs with their inclusive counterparts and testing models. Following [2], the evolution of the diffractive reduced cross section with $Q^{2}$ is compared with that of the inclusive DIS reduced cross section $\sigma_{r}$ by forming the ratio $\sigma_{r}^{D(3)}\left(x_{\mathbb{P}}, \beta, Q^{2}\right) / \sigma_{r}\left(x=\beta x_{\mathbb{P}}, Q^{2}\right)$ multiplied by $(1-\beta) x_{\mathbb{P}}$ at fixed $Q^{2}, \beta$ and $x_{\mathbb{P}}$, using a parameterisation of the $\sigma_{r}$ data from [5]. The diffractive reduced cross section is integrated over $|t|<1 \mathrm{GeV}^{2}$. The ratio of the diffractive to the inclusive cross section is indeed approximately constant with $\beta$ at fixed $Q^{2}$ except at large values of $x_{\mathbb{P}}$ where the sub-leading exchange contribution to the diffractive cross section is not negligible. In models in which both the diffractive and the inclusive cross sections are governed by a universal pomeron $[10,11]$, the remaining $\beta$ and $x_{\mathbb{P}}$ dependences of the ratio arises due to the deviations from unity of the pomeron trajectory and the contribution of the sub-leading trajectory. 
In order to compare the $Q^{2}$ dependences of the diffractive and the inclusive cross sections quantitatively, the derivative of their ratio with $\ln Q^{2}$ is extracted through fits of the form

$$
(1-\beta) x_{\mathbb{P}} \sigma_{r}^{D(3)}\left(x_{\mathbb{P}}, \beta, Q^{2}\right) / \sigma_{r}\left(x=\beta x_{\mathbb{P}}, Q^{2}\right)=a_{r}\left(\beta, x_{\mathbb{P}}\right)+b_{r}\left(\beta, x_{\mathbb{P}}\right) \cdot \ln Q^{2}
$$

The resulting values of $b_{r}$ are shown in figure 4. The ratio of the diffractive to the inclusive cross section depends only weakly on $Q^{2}$ for most $\beta$ and $x_{\mathbb{P}}$ values (the logarithmic derivative of the ratio, $b_{r}$, is consistent with zero within $1.5 \sigma$ of the experimental uncertainties). Whereas the diffractive and inclusive reduced cross sections are closely related to their respective quark densities, the $\ln Q^{2}$ derivatives are approximately proportional to the relevant gluon densities in regions where the $Q^{2}$ evolution is dominated by the $g \rightarrow \bar{q} q$ splitting. The compatibility of $b_{r}$ with zero thus implies that the ratio of the quark to the gluon density is similar in the diffractive and inclusive cases when considered at the same low $x$ values. Proton PDF predictions reproduce the behaviour of the $\ln Q^{2}$ derivative of the ratio with $\beta$.

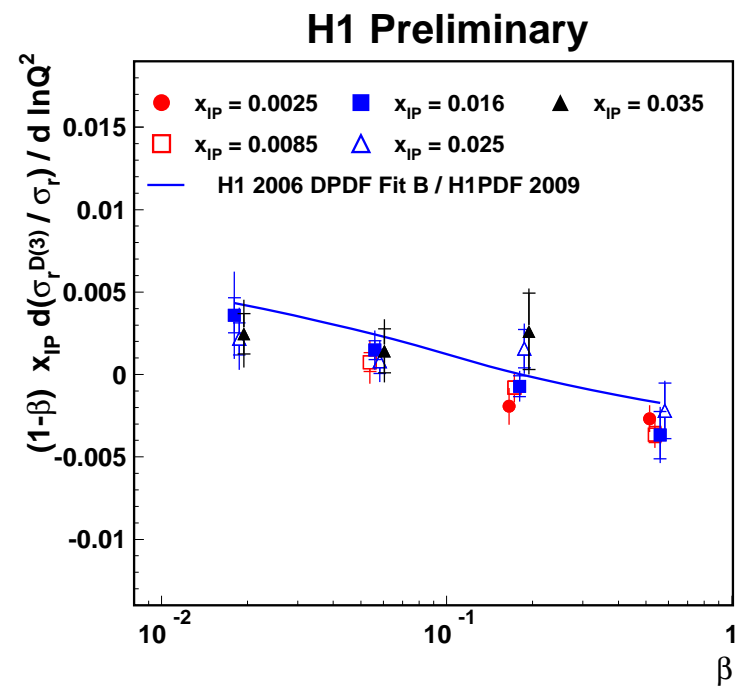

Figure 4: The logarithmic $Q^{2}$ derivative of the ratio of the diffractive reduced cross section $\sigma_{r}^{D(3)}\left(\beta, Q^{2}, x_{\mathbb{P}}\right)$ to the inclusive reduced cross section $\sigma_{r}\left(x=\beta x_{\mathbb{P}}, Q^{2}\right)$ multiplied by $(1-\beta) x_{\mathbb{P}}$, shown at different fixed values of $x_{\mathbb{P}}$ and $\beta$. The solid curve represents the results for the ratio of diffractive to inclusive PDF predictions [2,5].

\section{References}

[1] H1 Collaboration, Eur. Phys. J. C 48 (2006) 749

[2] H1 Collaboration, Eur. Phys. J. C 48 (2006) 715

[3] ZEUS Collaboration, Nucl. Phys. B 816 (2009) 1 [hep-ex/0408009].

[4] ZEUS Collaboration, Nucl. Phys. B 800 (2008) 1

[5] H1 Collaboration, Eur. Phys. J. C 64 (2009) 561 [arxiv:0904.3513]

[6] G. Jaroszkiewicz and P. Landshoff, Phys. Rev. D 10 (1974) 170.

[7] J. Bartels and H. Kowalski, Eur. Phys. J. C 19 (2001) 693 [hep-ph/0010345].

[8] J. Owens, Phys. Rev. D 30 (1984) 943.

[9] A. Mueller, Phys. Rev. D 2 (1970) 2963.

[10] K. Golec-Biernat and M. Wüsthoff, Phys. Rev. D 59 (1999) 014017 [hep-ph/9807513].

[11] J. Bartels, K. Golec-Biernat and H. Kowalski, Phys. Rev. D 66 (2002) 014001 [hep-ph/0203258]. 